\title{
Algoritmos RWA com Múltiplas Rotas em Redes Ópticas Dinâmicas
}

\author{
Victor A. P. Oliveira ${ }^{1}$ e Iguatemi E. Fonseca ${ }^{1}$
}

\begin{abstract}
Resumo - Este artigo apresenta um estudo sobre o impacto que o uso de múltiplas rotas tem sobre o desempenho de algoritmos de alocação de rota e de comprimento de onda em redes ópticas dinâmicas.
\end{abstract}

Palavras-chaves - Algoritmos RWA, Roteamento, Redes Ópticas Dinâmicas.

Abstract - This work presents a study of impact of the use of multiple routes on the performance of routing and wavelength assignment algorithm in dynamic optical networks.

Keywords - RWA algorithm, Routing, Dynamic Optical Networks.

\section{INTRODUÇÃO}

$\mathrm{R}$ edes ópticas surgiram originalmente a partir dos sistemas telefônicos, os quais formam projetados para transmitir circuitos de voz digitalizados através de uma fibra óptica (as chamadas Redes SONET/SDH) [1]. Com o crescimento acelerado de tráfego IP (Internet Protocol) e o surgimento e consolidação aplicações como vídeo sob demanda, voz sobre IP, etc., estas arquiteturas de rede estão sendo utilizadas para transportar tal tráfego. Entretanto, redes SONET/SDH não serão capazes de fluir o volume de tráfego no futuro (o qual espera-se ser mais intenso e dinâmico), pois empregam muito processamento eletrônico e, com isso, criam um "gargalo eletrônico". A utilização de processamento eletrônico também significa que redes SDH/SONET ainda são opacas, i.e., são baseadas em conexões ópticas terminadas em cada nó da rede por algum tipo de equipamento que realiza conversão eletro-óptica e vice-versa (O-E-O Optical-to-Electrical-to-Optical) [1].

Nesta direção, a área de comunicações ópticas, especificamente em redes, se situa hoje no centro dos esforços de construção de uma infraestrutura de comunicação adequada para a nascente Sociedade da Informação e a tecnologia IP sobre Divisão em Comprimento de Onda (WDM - Wavelength Division Multiplexing) parece ser uma estratégia interessante para resolver o problema do gargalo eletrônico [2]. Considerando as razões mencionadas acima, o problema geral abordado neste trabalho é estudar estratégias de roteamento que possam ser aplicadas aos algoritmos de alocação de rota e de comprimento de onda (RWA - Routing and Wavelength Assignment).

O restante deste artigo de TIC está organizado da seguinte forma. Na Seção II é descrito brevemente o funcionamento de

\footnotetext{
${ }^{1}$ Victor A. P. Oliveira e Iguatemi E. Fonseca, Departamento de Ciências Exatas e Naturais, UFERSA, RN, Brasil, Tel. +55-84-3315-1779, Email: victor_andre@uol.com.br, iguatemi@ufersa.edu.br, www.ufersa.edu.br/mcc. Os autores agradecem ao programa PIBIC/CNPq pelo apoio financeiro.
}

um algoritmo RWA típico e é apresentado a estratégia de roteamento estudada. Na seção III são discutidos os resultados das simulações numéricas e a Seção IV traz os comentários finais.

\section{Algoritmos RWA E RoteAmento}

\section{A. Algoritmo RWA}

O principal objetivo da rede óptica é dar suporte às suas redes clientes fornecendo-lhes caminhos ópticos, também chamados de conexões. O problema de estabelecer dinamicamente conexões em uma rede óptica envolve o uso de um algoritmo RWA [3]. Um caminho óptico é composto por uma sucessão de enlaces entre a origem e destino conectados por Nós Capazes de Fazer Roteamento (WRN Wavelength-Routing Node). Tradicionalmente, um caminho óptico é caracterizado pelo conjunto (rota, comprimento de onda) que são obtidos a partir da execução do algoritmo RWA na rede [4]. Por exemplo, como pode ser visto na Figura 1(a), o caminho óptico que liga os nós ‘b`e `f está acomodado no comprimento de onda $\lambda_{4}$.

\section{B. Roteamento com múltiplas rotas}

A Figura 2 traz o fluxograma de um algoritmo RWA estudado neste trabalho. Depois de gerada a requisição da conexão, de acordo com o estado atual da rede, o que configura um roteamento adaptativo [2], o algoritmo de Yen procura dentre todas as possibilidades as k-ésimas melhores rotas entre a origem e o destino [5]; o comprimento do enlace em $\mathrm{km}$ é considerado como custo pelo algoritmo de Yen. Após a execução do algoritmo de roteamento, elege-se portanto, dentre o conjunto total de rotas na rede, um subconjunto contendo as k rotas desejadas pelo cliente.

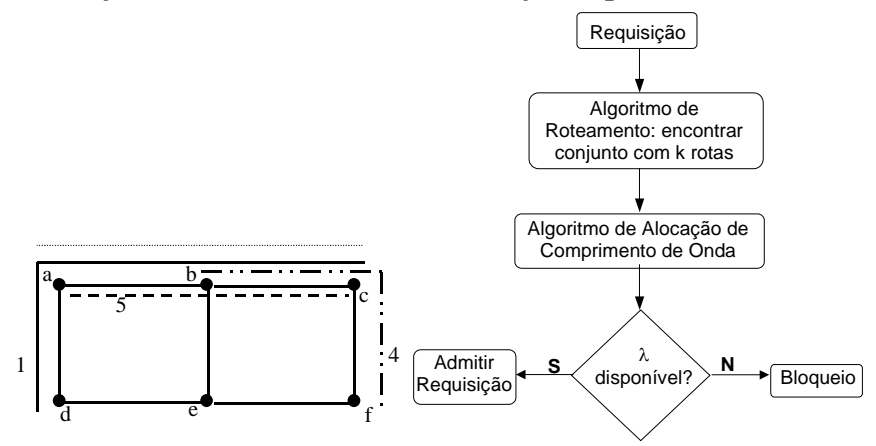

(a)

(b)

Fig. 1: (a) Rede óptica com três conexões ativas. (b) Fluxograma do Algoritmo RWA estudado.

O algoritmo de Yen, em essência, é um algoritmo usado para se achar as k-ésimas rotas mais curtas em uma dada rede, 
de acordo com algum custo definido para cada enlace. Na maioria de suas implementações utiliza-se o algoritmo de Dijkstra como parte do processo do algoritmo e ele possui complexidade $\mathrm{O}\left(\mathrm{kn}^{3}\right)$.

Depois de executado o algoritmo de roteamento, a disponibilidade comprimento de onda nas k-ésimas rotas candidatas é testada usando-se o algoritmo First-fit [6] e então se decide pela admissão ou bloqueio da requisição de conexão.

\section{SIMULAÇÕES NUMÉRICAS}

\section{A. Ambiente de Simulação}

Através de um ambiente de simulação estruturado, utilizando a linguagem de programação C, foi simulado um cenário dinâmico, no qual foram geradas 100000 requisições de conexões que possuem um padrão de tráfego uniforme entre os nós da rede e seguem uma distribuição poissoniana tendo duração com distribuição exponencial (média = 1s). A rede óptica utilizada é transparente, ou seja não possui conversão óptica-elétrica-óptica, e possui 26 nós, com todos os enlaces bidirecionais (ver topologia inserida nas Figuras 3 e 4). A partir desses parâmetros, a quantidade de conexões rejeitadas dentre o número total de pedidos de conexões que chegam na rede óptica é a Probabilidade de Bloqueio da rede. O número de saltos (hops) foi utilizado como custo dos enlaces no algoritmo de Yen.

\section{B. Resultados}

A Figura 3 traz a probabilidade de bloqueio versus tráfego em Erlang quando a rede opera com $\mathrm{W}=12$ comprimentos de onda e o algoritmo de roteamento busca as três melhores rotas. Perceba que o uso de uma estratégia simples de roteamento pode melhorar sensivelmente o desempenho da rede óptica, neste caso pode-se notar uma diminuição na probabilidade de bloqueio entre $10 \%$ e $20 \%$, dependendo do valor do tráfego. Por exemplo, para o tráfego de $80 \mathrm{Er}$, o roteamento com $\mathrm{k}=1$ (ou seja, somente a melhor rota) apresenta bloqueio de cerca de $10 \%$, contra cerca de $8 \%$ para o caso de roteamento com $\mathrm{k}=2$. Foi observado também que os resultados se repetem para o caso onde se usa um número menor de comprimentos de onda, como pode ser visto na Figura 4.

Quando se analisa o desempenho da estratégia de algoritmo estudada com relação ao tamanho das conexões na rede, verifica-se que o uso de múltiplas rotas também traz benefícios. A Figura 5 mostra a probabilidade de bloqueio versus tamanho da rota para conexões de tamanhos entre $1 \mathrm{e}$ 7 hops. Note que a medida que o tamanho da rota cresce, o algoritmo de roteamento com $\mathrm{k}=2$ e $\mathrm{k}=3$ rotas alternativas apresenta menor bloqueio que o roteamento com $\mathrm{k}=1$.

\section{COMENTÁRIOS FINAIS}

O uso de roteamento com múltiplas rotas se mostrou benéfico para o desempenho de redes ópticas dinâmicas. A inclusão de novas funcionalidades e estudo de algoritmos RWA com qualidade de serviço são os passos seguintes deste trabalho.

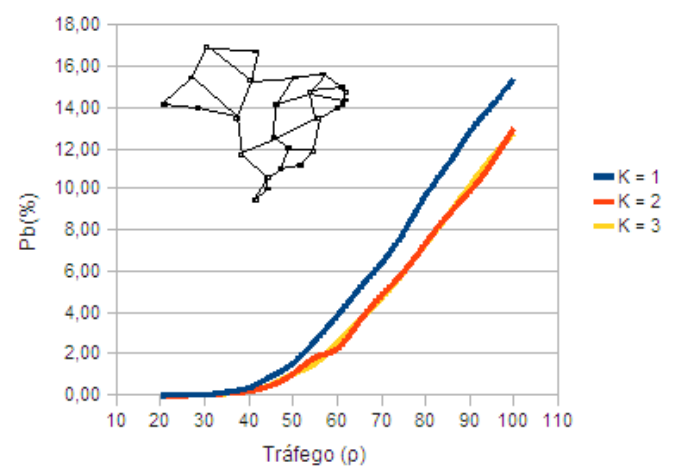

Fig. 3: Bloqueio para $\mathrm{W}=12$ comprimentos de onda.

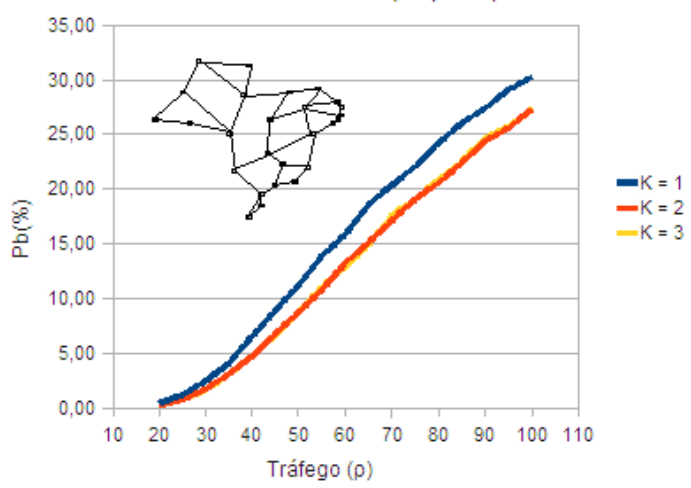

Fig. 4: Bloqueio para $\mathrm{W}=8$ comprimentos de onda.

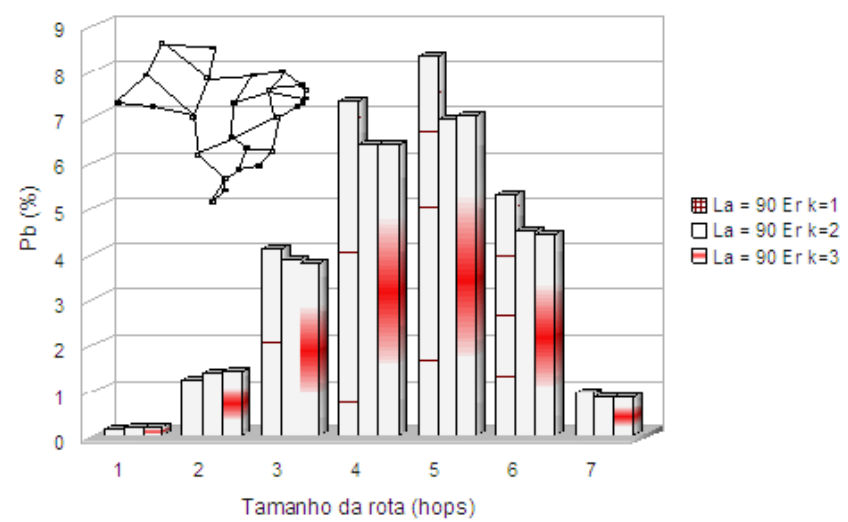

Fig. 5: Bloqueio por tamanho da rota para o tráfego de 90 Er.

\section{REFERÊNCIAS}

[1] R. Ramaswami, K.N. Sivarajan, “Optical networks: a practical perspective”, Academic Press, 2002.

[2] B. Mukherjee, “Optical WDM Networks”, Springer - optical networks series, 2006.

[3] R. Ramaswami, K.N Sivarajan,"Routing and Wavelength Assignment in All-optical Networks”, IEEE/ACM Transactions on Networking, Vol. 3, Oct 1995.

[4] H. Zang, J. P. Jue, and B. Mukherjee, "A Review of Routing and Wavelength Assignment Approaches for Wavelength-Routed Optical WDM Networks”, SPIE Optical Network Magazine, Vol. 1, pp. 47-60, Dec 2000.

[5] J. Yen, "Finding the $\mathrm{k}$ shortest loopless paths in a network", Management Science, vol. 17, pp. 712-716, July 1971.

[6] I. Chlamtac, A. Ganz, G. Karmi, "Lightpath communication: na approach to high bandwidth optical WAN's”, IEEE/ACM Transactions on Communications, Vol. 40, 1992. 